\title{
Enteropathy in Zambians with HIV related diarrhoea: regression modelling of potential determinants of mucosal damage
}

P Kelly, S E Davies, B Mandanda, A Veitch, G McPhail, I Zulu, F Drobniewski, D Fuchs, C Summerbell, N P Luo, J O M Pobee, M J G Farthing

\begin{abstract}
Background-AIDS is characterised by small intestinal mucosal damage, but its aetiopathogenesis is poorly understood. Enteric infections in Africa differ from those in northern countries, where protozoan infections have been associated with severe enteropathy in AIDS patients.

Aims-To characterise enteropathy in Zambian AIDS patients compared with local controls, and to assess relative contributions of enteric infection, nutritional impairment, and immune dysfunction.

Methods-Computer aided mucosal morphometry of small intestinal biopsy specimens from 56 HIV infected Zambians with persistent diarrhoea and 26 diarrhoea free controls, followed by regression modelling.

Results-Patients with HIV related diarrhoea had reduced villous height and increased crypt depth compared with controls. There was no difference between HIV positive and negative controls. In regression models applied to AIDS mucosal measurements, villous height and crypt depth were related to nutritional parameters and to serum soluble tumour necrosis factor receptor p55 concentration. Crypt depth was also related to lamina propria plasma cell count. Intestinal infection was found in $79 \%$, which consisted predominantly of microsporidia in $34 \%$, Isospora belli in $24 \%$, and Cryptosporidium parvum in $21 \%$, but detection of these enteropathogens was not related to severity of enteropathy.

Conclusions-Nutritional and immune disturbances were associated with enteropathy, accounting for over one third of the variation in mucosal morphometric parameters.

(Gut 1997; 41: 811-816)
\end{abstract}

Keywords: small intestine; enteropathy; protozoa; malnutrition; tumour necrosis factor receptors; Africa; AIDS

HIV infection is commonly accompanied by persistent diarrhoea and severe weight loss; this syndrome has been referred to as "slim disease" in sub-Saharan Africa. ${ }^{1}$ The aetiology of diarrhoea has not been clearly defined, but the protozoal enteropathogens found in African patients infected with $\mathrm{HIV}^{23}$ almost certainly play a part. Enteropathic changes in HIV disease in the small intestinal mucosa have been described in the developed world, ${ }^{4}$ and may be more severe in the tropics as HIV related enteropathic changes will be superimposed on background "tropical enteropathy". These changes probably reflect fundamental pathophysiological processes which may predispose to further intestinal infection, contribute to diarrhoea, and accelerate weight loss which may be important in determining survival. There are important differences between the infections prevalent in the developed world ${ }^{67}$ and those prevalent in Africa, ${ }^{8}$ where cytomegalovirus (CMV) ${ }^{9}$ and Mycobacterium avium complex infections are uncommon. ${ }^{10}$

The pathological processes leading to morphological damage in the small intestine are incompletely understood. Several noninfectious enteropathies are characterised by immune activation directed against the intestine: coeliac disease, ${ }^{11}$ transplant rejection, ${ }^{12}$ and graft versus host disease (GVHD). ${ }^{13}$ In vitro work indicates that in a fetal small intestine explant model, villous shortening can occur following $\mathrm{T}$ cell activation ${ }^{14}$ or HIV replication in the mucosa. ${ }^{15} \mathrm{HIV}$ is present in some immune cells of the gut and possibly in enterocytes, ${ }^{16}{ }^{17}$ but there is no evidence that AIDS is associated with mucosal $\mathrm{T}$ cell activation. ${ }^{18-20}$ Furthermore, one recent report found no difference in cytokine expression in the mucosa of AIDS patients with diarrhoea compared with healthy controls, ${ }^{21}$ but these patients did not seem to have advanced mucosal damage. Ultrastructural changes can occur in small intestinal mucosa of HIV infected individuals before the onset of opportunistic enteric infection. ${ }^{22}$

The role of opportunistic infection in HIV related enteropathy is unclear. Some workers have reported differences in mucosal morphology in patients with HIV related diarrhoea related to intestinal infection and those with "pathogen negative" diarrhoea. ${ }^{23}$ Similar findings were reported by Kotler $e t a l^{4}$ with respect to the presence of protozoa. New enteropathogens continue to be recognised and it may be that infections are more common than is generally appreciated. There is good evidence that cryptosporidiosis in immunocompetent children leads to villous atrophy, crypt hypertrophy, and disaccharidase deficiency, ${ }^{25}$ so it seems likely that Cryptosporidium parvum could damage the mucosa of immunocompromised
Accepted for publication 31 July 1997 
individuals. Recent work has shown that the severity of mucosal abnormalities in AIDS patients is related to the intensity of cryptosporidial infection, ${ }^{26}$ further supporting a pathogenetic role for this protozoan. Other infections such as $M$ avium complex, rotavirus, and giardiasis can also cause mucosal damage. The effect of malnutrition per se is unknown, but it is clear that the relationship between villous height, crypt depth, and intestinal infection in Africa will clearly be complex and may be different from that in temperate climates.

We analysed the relationship between intestinal mucosal morphology and potential determinants of enteropathy - namely enteric protozoal infection, mucosal inflammation, systemic immune activation, and undernutrition - using multivariate regression modelling in a cross sectional analysis of tissue from HIV seropositive African patients with persistent diarrhoea. We compared our findings in these patients with local controls to minimise the influence of tropical enteropathy.

\section{Patients and Methods}

PATIENTS

Fifty six HIV seropositive patients (30 males, 26 females; mean age 33 years, range 18-64) with persistent diarrhoea (of over three weeks duration) were recruited from the clinics and wards of the University Teaching Hospital, Lusaka. Six distal duodenal biopsy specimens were taken at random from the third part of the duodenum during upper gastrointestinal endoscopy under sedation. Nutritional assessment, as described in a previous report, ${ }^{27}$ involved measurement of weight, height, mid upper arm circumference (MUAC), and three skinfold thicknesses (triceps, subscapular, suprailiac) which were then added to obtain the total skinfold thickness (SFT). Skinfold thickness was measured using Holtain calipers. Anthropometric measurements were carried out by the same investigator (PK) on the day of endoscopy. Body mass index (BMI) was calculated as weight $/$ height $\left(\mathrm{kg} / \mathrm{m}^{2}\right)$.

Twenty six patients attending for endoscopy for dyspepsia were included as local controls. Nineteen of these patients underwent HIV testing and submitted one stool sample for examination for parasites, but in seven patients these investigations were not carried out. Nutritional assessment was not undertaken in the controls.

The study was approved by the Research Ethics Committee of the University of Zambia.

EVALUATION OF ENTEROPATHY USING LIGHT AND ELECTRON MICROSCOPY

One of the duodenal biopsy specimens from each patient was fixed in formal saline, processed to paraffin wax blocks, and serially sectioned to $5 \mu \mathrm{m}$. These sections were stained with haematoxylin and eosin, Giemsa, and Ziehl-Neelsen (ZN), and used for identification of parasites and acid-alcohol fast bacteria, and for morphometry. Morphometry was carried out with the Axio Home Graphics Workstation. ${ }^{28}$ Measurements were made of villous height $(\mathrm{VH})$, crypt depth (CD), villus: crypt ratio (VC), and total mucosal thickness (TMT) in biopsy specimens in which at least four well orientated villi were visible. Mucosal inflammation was quantified by counting the absolute number (total and differential) of chronic inflammatory cells in the lamina propria in an area defined by $300 \mu \mathrm{m}$ of muscularis mucosae. The intraepithelial lymphocyte count per 100 enterocytes (IEL) was also measured and expressed semiquantitatively as normal (less than 20 per 100 enterocytes), raised (20-40), or notably raised (over 40). Histological evaluation was carried out by an experienced pathologist (SED), who was blinded to the code used to identify specimens. Mucosal inflammation was quantified in all of the patients but in only seven of the controls.

A second duodenal biopsy specimen was fixed immediately in cacodylate buffered 3\% glutaraldehyde for 24 hours, and stored in $0.1 \mathrm{M}$ cacodylate buffer at $\mathrm{pH}$ 7.4. Specimens were treated with $1 \%$ osmium tetroxide and stained en bloc with saturated aqueous uranyl acetate before dehydration through a graded ethanol series. Finally, specimens were cleared in propylene oxide and embedded in epoxy resin. Semithin $(0.5 \mathrm{~mm})$ sections were mounted on slides and stained with toluidine blue in $1 \%$ borax; $60-90 \mathrm{~nm}$ sections were mounted on copper 300 mesh grids, stained with uranyl acetate and lead citrate, and examined in a Jeol JEM 100SX electron microscope. The electron microscopist (GMcP) was also blinded to the results of other investigations.

INVESTIGATION FOR ENTERIC INFECTION A stool specimen was collected from each patient on the day of endoscopy, and taken immediately to the laboratory. Up to three stool specimens were collected over several days, and each was examined by wet smear and by the modified Ziehl-Neelsen and modified trichrome stains. ${ }^{29}$ Faecal specimens were coded and then samples decontaminated using $4 \%$ sodium hydroxide and cultured in Kirchner broth and Lowenstein-Jensen medium with glycerol and pyruvate at $30^{\circ} \mathrm{C}$ for six months, with weekly examinations. Mycobacteria were identified by conventional methods. ${ }^{10}$

ASSAYS FOR MARKERS OF SYSTEMIC IMMUNE ACTIVATION

Serum was collected on the day of endoscopy and stored at $-20^{\circ} \mathrm{C}$. Serum neopterin was assayed by radioimmunoassay and serum soluble tumour necrosis factor receptors type I (sTNF-R55) and type II (sTNF-R75) were assayed by enzyme linked immunological and biological binding assay (ELIBA). ${ }^{30}$

\section{STATISTICAL ANALYSIS}

Statistical analysis was carried out using Epi-info version 6.0 (WHO and CDC, Atlanta). Comparisons were made using Fisher's exact or Kruskal-Wallis tests as some data displayed a skewed distribution (see later). Linear correlation was expressed as $r$ with $95 \%$ confidence intervals (CI). Multiple linear regression was performed using age, duration and 
TABLE 1 Clinical characteristics of 56 patients with HIV related persistent diarrhoea

\begin{tabular}{lcc}
\hline Parameter & Mean & Range \\
\hline Age (years) & 33.1 & $18-64$ \\
Male:female & $30: 26$ & \\
Duration of diarrhoea (months) & 9.6 & $1-48$ \\
Tuberculosis diagnosed (lifetime) & $15(27 \%)$ & \\
BMI (kg/m $\left.{ }^{2}\right)$ & 15.6 & $11-22$ \\
MUAC & 20.9 & $15-28$ \\
SFT & 17.2 & $9-31$ \\
Fever at time of study & $24(43 \%)$ & \\
\hline
\end{tabular}

Reference ranges for values of BMI, MUAC and SFT are discussed by Kelly et al. $^{27}$

frequency of diarrhoea, nutritional status (BMI, MUAC, SFT, serum albumin), serum neopterin, sTNF-R75 and sTNF-R55 concentrations, IEL count, and total and differential lamina propria cell counts as potential correlates, using a backwards stepwise regression strategy and elimination of non-contributory terms at each step according to the partial F-test, 95\% CI for the regression coefficient, and proportion of variability explained. In each final model only one measure of nutritional status was allowed. As the distribution of
VH

CD

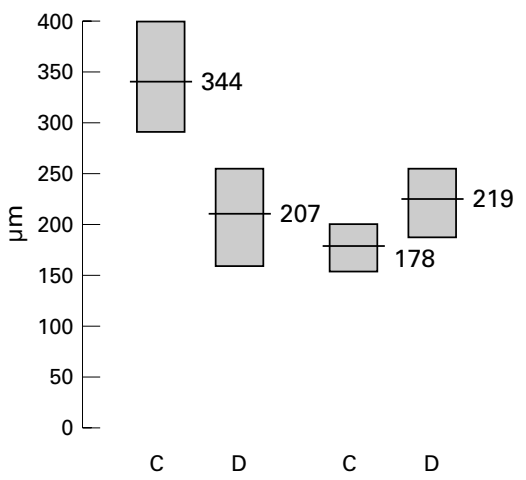

VH:CD ratio

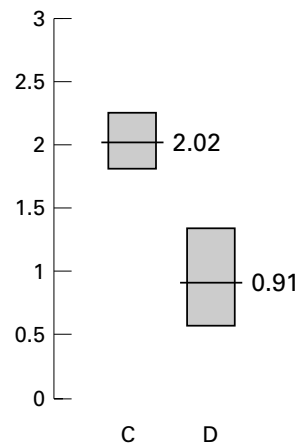

Figure 1: Villous height (VH), crypt depth (CD), and VH:CD ratio in 56 patients with HIV related diarrhoea (D) and 26 diarrhoea free controls attending for endoscopy for dyspepsia (C). Horizontal bars represent median values, and shaded boxes interquartile range. The differences between patients and controls were significant using the Kruskal-Wallis test $(p<0.001$ for all three parameters).

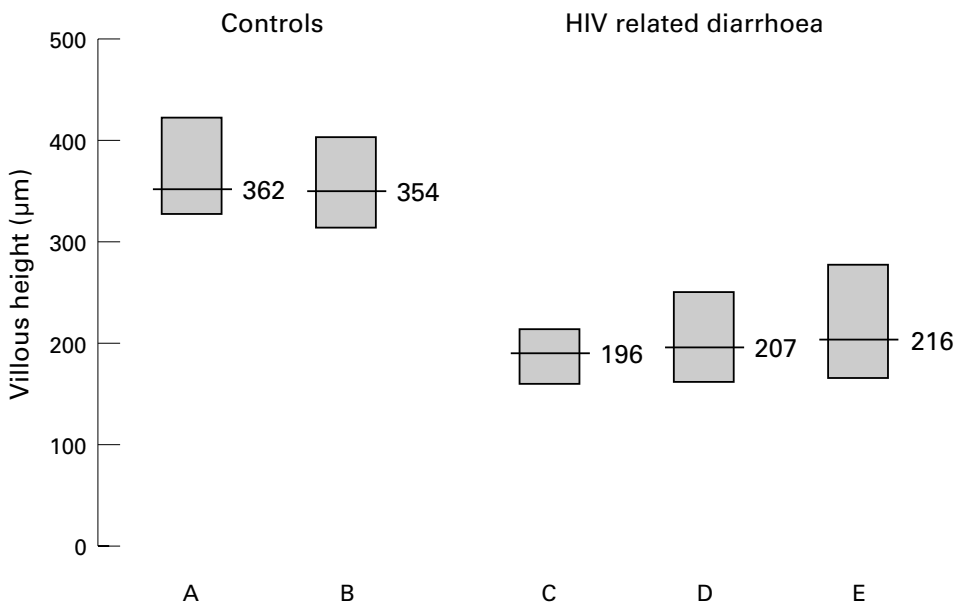

Figure 2: Villous height (VH) in different groups of subjects. In seven controls who were HIV positive (A), VH did not differ from that of $12 \mathrm{HIV}$ negative controls (B). Within the group of patients with HIV related persistent diarrhoea, $V H$ did not differ in 21 patients with small intestinal protozoal infection seen by light or electron microscopy $(C), 21$ patients with protozoal infection but in whom no small intestinal infection was detected (D), and 14 patients with no infection detected at all (E). Crypt depth also did not differ except between patients with diarrhoea and controls (fig 1). serum neopterin and sTNF-R55 concentrations was skewed, the analysis was repeated using $\log$ transformations of these variables; this made no significant difference to the final model or to the regression parameters.

\section{Results}

CLINICAL AND ENDOSCOPIC FINDINGS

The patients were wasted, with advanced HIV disease (table 1). Of the 56 patients with persistent diarrhoea, 52 (93\%) had AIDS (stage IVc) defining conditions. Oesophageal candidiasis was present in $33(59 \%)$ patients. Gastric Kaposi sarcoma was present in two patients and gastric erosions in four.

ENTEROPATHY IN DISTAL DUODENAL MUCOSA Distal duodenal biopsy specimens from patients with persistent diarrhoea showed marked enteropathic changes compared with the local controls (fig 1), but HIV positive and HIV negative controls did not differ (fig 2). Villous height was reduced and crypt depth increased, and these changes were mirrored in the villous height:crypt depth ratio. These villus and crypt architectural changes in biopsy specimens from patients with diarrhoea were accompanied by mucosal inflammation. Lamina propria total cell count was non-significantly increased compared with controls, but not for differential counts (table 2). IEL counts were increased in patients with diarrhoea compared with controls: $44 / 56(78 \%)$ had IEL counts over 20 per 100 enterocytes compared with none of the controls. Five of the eight specimens which had a heavily eosinophilic infiltrate were from patients with isosporiasis ( $p=0.01$ by Fisher's exact test), but otherwise total and differential lamina propria cell counts did not differ between patients with different infections and those apparently "pathogen negative".

Electron microscopy showed the presence of parasites in $20(36 \%)$ biopsy specimens. None of the specimens studied had any evidence of viruses. Electron microscopy also showed enterocyte changes, including microvillous loss (25\%), mitochondrial swelling (39\%), formation of lysosomal and vesicular bodies (93\%), and dilatation of the endoplasmic reticulum $(70 \%)$. Enterocytes did not appear flattened. There was no association between these ultrastructural changes and villous or crypt parameters, lamina propria inflammation, or the presence of protozoa in the biopsy specimen.

\section{INTESTINAL INFECTION}

All 58 patients provided at least one stool for examination, $42(72 \%)$ provided two, and 21 $(36 \%)$ provided three. The cumulative yield of stool microscopy was $38 \%$ after one examination, $45 \%$ after two examinations, and $50 \%$ after three examinations. Infections were found in $79 \%$ of patients (table 3), predominantly microsporidia, Isospora belli, and $C$ parvum. No oocysts of Cyclospora spp. were found. Mycobacteria were cultured from six patients: two had $M$ avium complex, and four $M$ tuberculosis, ${ }^{10}$ but none of the duodenal biopsy specimens 
TABLE 2 Lamina propria cell counts in duodenal biopsy specimens

\begin{tabular}{llll}
\hline & Controls $(n=7)$ & $\begin{array}{l}\text { Diarrhoea } \\
(n=56)\end{array}$ & $p$ Value \\
\hline Cell count (total) & $233(144-299)$ & $285(173-443)$ & 0.07 \\
Lymphocytes & $105(23-186)$ & $124(38-307)$ & 0.55 \\
Plasma cells & $106(71-141)$ & $147(40-317)$ & 0.11 \\
Eosinophils & $21(11-31)$ & $15(0-83)$ & 0.05 \\
\hline
\end{tabular}

Mean total and differential cell counts in that area of lamina propria defined by $300 \mu \mathrm{m}$ muscularis mucosae in biopsy specimens from 56 patients with HIV related diarrhoea and from seven controls (HIV status unknown). The Kruskal-Wallis nonparametric test was used to calculate the $\mathrm{p}$ values.

TABLE 3 Intestinal infection in 56 patients with HIV related diarrhoea in Lusaka

\begin{tabular}{ll}
\hline Pathogen & Number (\%) \\
\hline Microsporidia $^{a}$ & $20(34)$ \\
Isospora belli $_{\text {Cryptosporidium parvum }}$ & $14(24)$ \\
Blastocystis hominis $^{b}$ & $12(21)$ \\
Strongyloides stercoralis & $6(10)$ \\
Giardia intestinalis $_{\text {Mycobacterium tuberculosis }}$ & $1(1)$ \\
Mavium complex & $4(7)$ \\
Negative & $2(3)$ \\
\end{tabular}

${ }^{a}$ Species identified by electron microscopy in seven of these: five Enterocytozoon bieneusi, two Septata intestinalis.

${ }^{\mathrm{b}}$ The status of $B$ hominis as a pathogen is controversial.

showed the infiltrative pattern commonly seen in North American or European patients. Fungal spores were present in $60 \%$ of stool samples.

RELATIONSHIP OF ENTEROPATHY TO POTENTIAL DETERMINANTS: UNIVARIATE ANALYSIS

Villous height, crypt depth, and total mucosal thickness did not differ in patients with intestinal infections and in those who were apparently "pathogen negative" (fig 3). In addition, there was no morphological difference between biopsy specimens from patients found (by electron microscopy) to have duodenal infections and those with (presumably) more distal infections (fig 2), nor between patients with single or multiple infections. Age, sex, duration of diarrhoea, frequency of diarrhoea, and endoscopic findings were not related to the severity of mucosal abnormalities.

Neopterin and sTNF-R concentrations in serum were shown in a previous report to be considerably elevated in this cohort of patients
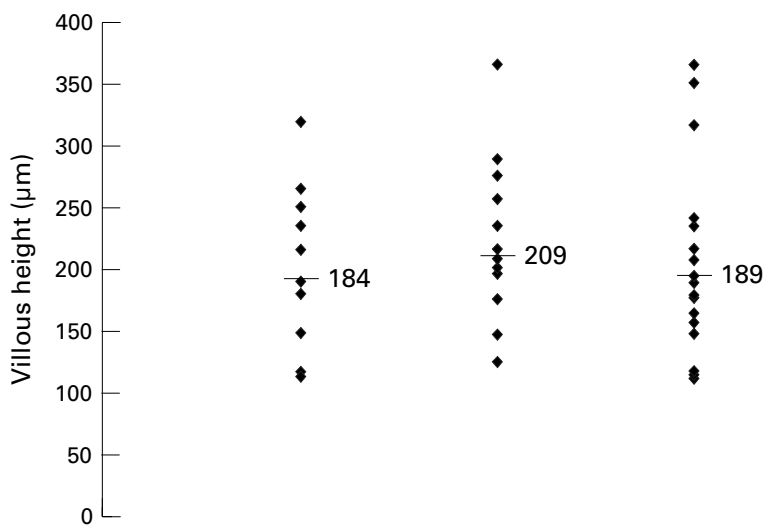

Cryptosporidium

Isospora

Microsporidia

None

Figure 3: Villous height in patients with different intestinal infections or who were apparently "pathogen negative". No differences were seen between the major diagnostic groups. Crypt depth also showed no differences.
TABLE 4 Relation of mucosal morphology to measures of nutritional status and to $S T N F-R 55$ concentration

(univariate analysis) in 56 patients with HIV related diarrhoea

\begin{tabular}{lll}
\hline Nutritional measure & $r$ & $95 \% \mathrm{CI}$ \\
\hline Villous height & & \\
BMI & 0.19 & $\mathrm{NS}$ \\
MUAC & 0.30 & $0.04,0.52$ \\
SFT & 0.38 & $0.09,0.60$ \\
$\quad$ sTNF-R55 & -0.32 & $-0.54,-0.06$ \\
Mucosal thickness & & \\
BMI & 0.40 & $0.15,0.60$ \\
MUAC & 0.40 & $0.15,0.60$ \\
SFT & 0.37 & $0.08,0.59$ \\
sTNF-R55 & -0.45 & $-0.63,-0.22$ \\
Crypt depth & & \\
BMI & 0.30 & $0.05,0.52$ \\
MUAC & 0.17 & $\mathrm{NS}$ \\
SFT & 0.03 & $\mathrm{NS}$ \\
sTNF-R55 & -0.30 & $-0.52,-0.04$ \\
\hline
\end{tabular}

Linear correlation coefficients for nutritional parameters and sTNF-R55 concentration in univariate analysis of $\mathrm{VH}, \mathrm{CD}$ and TMT. $r$ is shown, together with $95 \%$ confidence intervals (CI). NS indicates that the $95 \%$ confidence intervals include zero.

with persistent diarrhoea compared with diarrhoea free controls. ${ }^{27}$ Both sTNF-R55 and sTNF-R75 concentrations were elevated and the same pattern was observed for both molecules; only sTNF-R55 has been shown in the analyses as the spread of values was slightly greater. sTNF-R55 concentrations were not significantly higher in febrile compared with non-febrile subjects. VH, CD, and TMT were correlated with sTNF-R55 concentrations and nutritional status (table 4), but not serum neopterin concentrations.

RELATIONSHIP OF ENTEROPATHY TO POTENTIAL DETERMINANTS: MULTIVARIATE REGRESSION ANALYSIS

Best fit multiple linear regression equations were:

(1) $\mathrm{VH}=187+(2.0 \times$ serum albu$\min )-(4.45 \times \mathrm{s}$ TNF-R55)

$(F=6.46$, p $<0.01$; proportion of variability in $\mathrm{VH}$ accounted for: $25 \%$ )

(2) $\mathrm{TMT}=426+(4.45 \times \mathrm{SFT})-(11.43 \times$ sTNF-R55)

$(F=11.93, \mathrm{p}<0.001$; proportion of variability in TMT accounted for: $36 \%$ )

(3) $\mathrm{CD}=64+(7.9 \times \mathrm{BMI})+(0.36 \times$ lamina propria plasma cell density $)-(2.57 \times \mathrm{sTNF}$ R55)

$(F=8.51, \mathrm{p}<0.001$; proportion of variability in CD accounted for: $33 \%$ )

$\mathrm{VH}$ and TMT were thus positively related to measures of nutritional status and negatively related to sTNF-R55 concentration. In both equations (1) and (2), serum albumin concentration and total skin fold thickness (SFT) were almost interchangeable. Crypt depth was positively related both to lamina propria plasma cell density and to $\mathrm{BMI}$, and negatively to sTNF-R55 concentration. sTNF-R55 and sTNF-R75 concentrations could also be interchanged in these equations.

\section{Discussion}

These AIDS patients in Zambia with persistent diarrhoea had marked enteropathy, and enteric infections, often multiple, were detected in a 
high proportion. There was no difference in mucosal morphometry between HIV seropositive and HIV seronegative local controls. There was no difference in the severity of enteropathy in patients with different infections, but few patients were found without detectable infection, and many infections were multiple, possibly obscuring any differences which might exist. The multiple regression models also indicated that enteropathy was strongly related to nutritional status and to immune dysregulation as revealed by serum sTNF-R55 concentrations, which we have previously reported to be high. ${ }^{27}$ The enteropathic changes were quite pronounced, with a $40 \%$ reduction in mean villous height and $19 \%$ increase in mean crypt depth. These changes cannot be explained by tropical enteropathy as the morphometric parameters have been compared with local controls. The villous heights found in the control subjects here were slightly greater than those found in a study of Afro-Caribbean immigrants to the UK. ${ }^{31}$ Crypt depth was increased in patients with diarrhoea, but we note that it was positively related to nutritional status (BMI in this instance) and negatively to serum sTNF-R55 concentrations. This might signify that increased crypt depth is a response to injury which is impaired in the presence of a nutritional deficit.

Mucosal morphometry carried out in this way is subject to error, because of inadequacies in the process of fixation and orientation which mean that it is difficult to ensure that the entire length of the villus can be measured. Furthermore, one or two biopsy specimens from the distal duodenum may not represent adequately the whole of the small intestinal mucosa. We therefore analysed villous height and crypt depth to look for evidence that infections detected in these biopsy specimens might influence enteropathy, and found none. Likewise, anthropometry at a single time point is a crude method of assessment of body composition, and fails to reflect rates of change. Given these shortcomings, it is all the more notable that the regression models were able to account for appreciable fractions of variability in mucosal parameters.

There was no evidence that intestinal protozoal infection influenced the severity of enteropathy, irrespective of: the species of infecting parasite; the presence of the parasite in the same part of the small intestine as the biopsy specimens were collected; or the presence of multiple infections. This finding is at variance with previous studies which observed some relationship between enteropathy and protozoan infection, ${ }^{23}{ }^{24}$ but supports the work of Greenson $e t a l .{ }^{32}$ As protozoa were found in the majority $(79 \%)$ of the patients in this study, who had quite advanced HIV disease, it is distinctly possible that multiple infections were more common than was apparent given the insensitivity of most microscopic detection techniques. $C$ parvum is known to damage the mucosa in immunocompetent children ${ }^{25}$ and it would be surprising if it had no such effect in the immunodeficient. Other infections are also associated with enteropathic changes, particu- larly $M$ avium complex. Stool cultures for salmonellae, shigellae, and Campylobacter spp. were not undertaken, nor were virological examinations of stool. However, no viral particles were seen by electron microscopic examination of the biopsy specimens, and other studies have indicated that viral infections do not play a major role in this syndrome. ${ }^{33}$ Salmonella and Shigella spp. were not found in Zambian patients in the study of Conlon et $a l^{3}$ and facilities for Campylobacter isolation were not available at the time of the study. Colonic biopsy specimens were not taken as colonic infection seemed a priori to be unlikely to affect mucosal morphology in the proximal small intestine.

These regression models clearly indicate that nutritional parameters are related to mucosal morphometric measures. There is little direct evidence in humans that structural intestinal damage follows from wasting of body mass in the absence of enteric or systemic infection, but it is established in animals. ${ }^{34}$ Starvation in humans can bring about enteric dysfunction, manifest as increased secretion of water and electrolytes. $^{35}$

sTNF-R concentrations in serum were also found to be related to mucosal change, but this was not true of neopterin concentrations. sTNF-R55 and neopterin concentrations in serum probably reflect $\mathrm{TH}_{1}$ lymphocyte activation by HIV or by opportunistic pathogens and/or macrophage directed activation by opportunistic pathogens or lipopolysaccharide. sTNF-R concentration is linearly related to TNF- $\alpha$ concentration ${ }^{36}$ and the former was therefore used in this study as free TNF can be difficult to measure in the peripheral circulation because it is rapidly cleared. It is well established that markers of immune activation act as indicators of progression of HIV disease, but much more work is required to define the precise molecular and cellular basis of these disturbances. Individual serum cytokine assays, if sufficiently sensitive tests of a large range of such molecules were available, would help clarify the mechanism of disturbance. There is little support for the hypothesis that mucosal $\mathrm{T}$ cell activation occurs in HIV related enteropathy, ${ }^{18}{ }^{19}$ and in a subset of the patients reported here, no evidence of mucosal $\mathrm{T}$ cell activation was found. ${ }^{20}$ Unfortunately, when this study was conducted, CD4 measurements were not available in this institution. Such measurements might have contributed usefully to understanding the potential determinants of enteropathy.

What is the significance of enteropathy in AIDS? Enteropathy itself does not directly lead to diarrhoea, as it is not uncommon to find patients with coeliac disease and subtotal villous atrophy who have normal bowel habit, and this may depend on the extent of small intestinal damage. ${ }^{37}$ However, the alteration in balance between villous (absorptive) and crypt (secretory) cell populations may predispose to salt and water malabsorption and diarrhoea will then ensue if colonic water absorption is impaired. Although enteropathy does not directly lead to weight loss (which is largely 
attributable to reduced calorie intake ${ }^{38}$ ) through malabsorption of calories, enteropathy may contribute to micronutrient malabsorption.

The small intestinal mucosa in these patients appears to have changed in response to a complex array of nutritional and immunological insults, although its relationship to current or previous enteric infections is difficult to understand. Better understanding of the mechanism of mucosal damage will probably require observing the response to eradication of protozoa and further study of these immunological abnormalities.

We are grateful for the financial support of the Wellcome Trust, the Smith and Nephew Research Foundation, the Association of Physicians of Great Britain and Ireland, and Keymed Ltd. We would like to acknowledge the dedicated work of Mrs S Mwanamakondo and Ms R Chileshe in the UTH endoscopy unit.

1 Serwadda D, Mugerwa RD, Sewankambo NK, Lwegaba A Carswell J, Kirya GB. Slim disease: a new disease in Uganda and its association with HTLV III infection. Lance 1985;ii:849-52.

2 Colebunders R, Lusakumuni K, Nelson AM, Gigase P, Lebughe I, Van Marck E. Persistent diarrhoea in Zairian AIDS patients: an endoscopic and histological study. Gut 1988;29:1687-91.

3 Conlon C, Pinching AP, Perera CU, Moody A, Luo NP, Lucas SB. HIV related enteropathy in Zambia: a clinical, microbiological and histological study. Am F Trop Med Hyg 1990;42:83-8.

4 Batman PA, Miller ARO, Forster SM, Harris JRW, Pinching AJ, Griffin GE. Jejunal enteropathy associated with HIV infection: quantitative histology. F Clin Pathol 1989;42:275infection 81 .

5 Cook GC. Tropical gastroenterology. Oxford: Oxford University Press, 1980.

6 Rene E, Marche C, Regnier B, Saimot AG, Vilde JL, Perrone $\mathrm{C}$, et al. Intestinal infection in patients with AIDS A prospective study in 132 patients. Dig Dis Sci 1989;34:773-80

7 Smith PD, Lane HC, Gill VJ, Manishewitz JF, Quinnan GV, Fauci AS, Masur H. Intestinal infections in patients with AIDS. Ann Intern Med 1988;108:328-33.

8 Lucas SB. Missing infections in AIDS. Trans $R$ Soc Trop Med Hyg 1990;84(suppl 1):S34-8.

9 Colebunders RL, Latif AS. Natural history and clinical presentation of HIV-1 infection in adults. AIDS 1991; 5(suppl 1):S103-12.

10 Pankhurst CL, Luo N, Kelly P, Drobniewski F, Ngwenya B, Farthing MJG. Intestinal mycobacteria in African AIDS patients. Lancet 1995;345:585.

11 Trejdosiewicz LK, Howdle PD. T cell responses and cellular immunity. Baillieres Clin Gastroenterol 1995;9:251-72.

12 Lee RG, Nakamura K, Tsamandas A, Abu-Elmagd K, Furukawa $\mathrm{H}$, Hutson WR, et al. Pathology of human intesFurukawa H, Hutson WR, et al. Pathology of human intes

13 Mowat AMcI, Felstein MV. Experimental studies of immunologically mediated enteropathy V. Destructive enteropathy during and acute graft-versus-host reaction in adul BDF1 mice. Clin Exp Immunol 1990;79:279-84.

14 Lionetti P, Breese E, Braegger CP, Murch SH, Taylor J, MacDonald TT. T cell activation can induce either mucosal destruction or adaptation in cultured human fetal small intestine. Gastroenterology 1993;105:373-81.

15 Batman PA, Fleming SC, Sedwick PM, MacDonald TT, Griffin GE. HIV infection of human fetal intestinal explant cultures induces epithelial cell proliferation. AIDS $1994 ; 8$ 161-7.

16 Fox CH, Kotler D, Tierney A, Wilson C, Fauci AS. Detection of HIV-1 RNA in the lamina propria of patients with AIDS and gastrointestinal disease. F Infect Dis 1989;159: 467-71.
17 Heise C, Dandekar S, Kumar P, DuPlantier R, Donovan RM, Halsted CH. HIV infection of enterocytes and mononuclear cells in human jejunal mucosa. Gastroenterology 1991;100:1521-7.

18 Lim SG, MacDonald TT, Francis N, Gazzard BG. Alterations in mucosal immune cells in HIV positive patients with duodenal cryptosporidiosis [abstract]. Gastroenterology 1994;106:A721.

19 Ullrich R, Zeitz M, Heise W, L'Age M, Ziegler K, Bergs C, Riecken EO. Mucosal atrophy is associated with loss of activated T cells in the duodenal mucosa of HIV-infected patients. Digestion 1990;46:S302-7.

20 Veitch AM, Kelly MP, Luo N, Pobee JOM, Penney LS, Farthing MJG. T cell activation in small intestinal mucosa of African AIDS patients with chronic diarrhoea [abstract]. Gut 1995;36:A54

21 Snijders F, van Deventer SJH, Bartelsman JFW, den Otter P, Jansen J, Mevissen MLCM, et al. Diarrhoea in HIVinfected patients: no evidence of cytokine-mediated inflammation in jejunal mucosa. AIDS 1995;9:367-73.

22 Mathan MM, Griffin GE, Miller A, Batman P, Forster S, Pinching A, Harris W. Ultrastructure of the jejunal mucosa in HIV infection. F Pathol 1990;161:119-27.

23 Ullrich R, Zeitz M, Heise W, L'age M, Hoffken G, Riecken EO. Small intestinal structure and function in patients infected with HIV: evidence for HIV induced enteropathy. Ann Intern Med 1989;111:15-21.

24 Kotler DP, Francisco A, Clayton F, Scholes JV, Orenstein JM. Small intestinal injury and parasitic diseases in AIDS. Ann Intern Med 1990;113:444-9.

25 Phillips AD, Thomas AG, Walker Smith JA. Cryptosporidium, chronic diarrhoea, and the proximal small intestinal mucosa. Gut 1992;33:1057-61.

26 Genta RM, Chappell CL, White AC, Kimball KT, Goodgame RW. Duodenal morphology and intensity of infection in AIDS-related intestinal cryptosporidiosis. Gastroenterology 1993;105:1769-75

27 Kelly P, Summerbell C, Ngwenya B, Mandanda B, Hosp M, Luo $\mathrm{N}$, et al. Systemic immune activation as a determinant of wasting in patients with AIDS and diarrhoea in Zambia. Of Med 1996;89:831-7.

28 Slavin G, Sowter C, Robertson K, McDermott S, Paton K. Measurement in jejunal biopsies by computer-aided microscopy. $f$ Clin Pathol 1980;33:254-61.

29 Drobniewski F, Kelly P, Karew A, Ngwenya B, Luo N, Pankhurst C, Farthing M. Human microsporidiosis in African AIDS patients with chronic diarrhoea. $\mathcal{f}$ Infect Dis 1995;171:515-6.

30 Zangerle R, Gallati H, Sarcletti M, Weiss G, Denz H, Wachter H, Fuchs D. Increased serum concentrations of soluble tumour necrosis factor receptors in HIV-1 infected individuals are associated with immune activation. $f$ Acquir Immune Defic Syndr 1994;7:79-85.

31 Wood GM, Gearty JC, Cooper BT. Small bowel morphology in British and Afro-Caribbean subjects: evidence of tropical enteropathy. Gut 1991;32:256-9.

32 Greenson JK, Belitsos PC, Yardley JH, Bartlett JG. AIDS enteropathy: occult enteric infections and duodenal mucosal alterations in chronic diarrhea. Ann Intern Med 1991; 114:366-72

33 Thea DM, Glass R, Grohmann GS, Perriens J, Ngoy B, Kapita B, et al. Prevalence of enteric viruses among hospitalised AIDS patients in Kinshasa, Zaire. Trans $R$ Soc Trop Med Hyg 1993;87:263-6.

34 Zambonino-Infante JL, Rouanet JM, Besancon P. Mathematical correlation between villus height and the nutritional state in Sprague-Dawley rats. Gut 1993;34:1066-8.

35 Young A, Levin RJ. Diarrhoea of famine and malnutrition: investigations using a rat model. Gut 1990;31:43-53,162-9.

36 Grimble RF. Interaction between nutrients, pro-inflammatory cytokines and inflammation. Clin Sci 1996;91:12130 .

37 Marsh MN, Crowe PT. Morphology of the mucosal lesion in gluten sensitivity. Baillieres Clin Gastroenterol 1995;9: 273-93.

38 Macallan DC, Noble C, Baldwin C, Jebb SA, Prentice AM, Coward A, et al. Energy expenditure and wasting in HIV infection. $N$ Engl f Med 1995;333:83-8. 